\title{
Las estrategias de afrontamiento frente a la percepción de apoyo social: Estudio Descriptivo-Comparativo con adultos mayores institucionalizados y no institucionalizados de la Región Metropolitana, considerando la variable género. ${ }^{1}$
}

\section{Strategies for Coping with Perceptions of Social Support: a Descriptive-Comparative Study with Senior Citizens in Care Homes and in Private Homes in the Metropolitan Region, Considering Gender Differences}

\author{
Carolina Reyes Rasse \\ Paz Rojas Arellano \\ Universidad Santo Tomás de Chile
}

\begin{abstract}
La presente investigación estudió las estrategias de afrontamiento frente a la percepción de apoyo social en adultos mayores institucionalizados y no institucionalizados, considerando la variable género. Se utilizó una metodología de tipo descriptivo-comparativo de carácter cuantitativo.

La muestra comprendió 100 adultos mayores, 25 hombres institucionalizados y no institucionalizados, 25 mujeres institucionalizadas y no institucionalizadas.

Los resultados señalan que las mujeres adultas mayores perciben mayor apoyo social familiar y de las amistades que los hombres; de igual manera, los no institucionalizados perciben mayor apoyo social que los institucionalizados. Comparando ambas escalas de apoyo social, los adultos mayores perciben mayor apoyo familiar que de las amistades.

Con respecto a las estrategias de afrontamiento, entre hombres y mujeres adultos mayores existen diferencias significativas en la utilización de las estrategias: reinterpretación positiva y crecimiento, focalización y ventilación de emociones, siendo las mujeres quienes las despliegan más. Los hombres utilizan más las estrategias: afrontamiento activo, aceptación de responsabilidad y autocontrol, y las mujeres reinterpretación positiva y crecimiento, aceptación de responsabilidad y autocontrol.

Los adultos mayores institucionalizados despliegan menos estrategias de afrontamiento que los no institucionalizados, existiendo diferencias significativas en las estrategias: planificación, búsqueda de apoyo social por motivos instrumentales, búsqueda de apoyo social por motivos afectivos y reinterpretación positiva y crecimiento. Las principales estrategias utilizadas por los adultos mayores institucionalizados son: afrontamiento activo, aceptación de la responsabilidad y autocontrol. Los no institucionalizados utilizan más las estrategias: afrontamiento activo, planificación, reinterpretación positiva y crecimiento y de autocontrol. Palabras clave: Estrategias de afrontamiento, percepción de apoyo social, adulto mayor
\end{abstract}

This research studied the strategies for dealing with social support as perceived by senior citizens in and outside of institutions, considering gender differences. A quantitative, descriptive-comparative methodology was used.

The sampling was comprised of 100 senior citizens, 25 men in and outside of institutions, and 25 women in and outside of institutions.

The results show that older women perceive more social support from family and friends than do men, and that those living in private homes perceive greater social support than do those in care homes. In a comparison of both social support scales, the elderly perceive greater support from family than from friends.

With respect to strategies for coping with the situation, there are significant differences between how elderly men and women use the strategies of positive reinterpretation and growth, focalizing and expressing emotions; the women are more likely to deploy these strategies. Men are more likely to use the strategies of actively coping with the situation, accepting responsibility, and self-control, while women use positive reinterpretation and growth, accepting responsibility, and self-control.

The elderly in care homes deploy fewer strategies than those outside of institutions, with major differences in the strategies used: planning, seeking social support for practical reasons, seeking support for emotional reasons, and positive reinterpretation and growth. The main strategies used by senior citizens in care homes are: actively coping with the situation, accepting responsibility, and self-control. The elderly at home tend to use the strategies of actively coping with the situation, planning, positive reinterpretation and growth, and self-control.

Key words: Coping strategies, perception of social support, senior citizen 
El objetivo general de esta tesis consiste en describir y comparar las estrategias de afrontamiento utilizadas por adultos mayores institucionalizados y no institucionalizados frente a la percepción de apoyo social, considerando la variable sociodemográfica género en la Región Metropolitana.

Como objetivos específicos se señalan:

1. Describir la percepción de apoyo social familiar y de las amistades en adultos mayores institucionalizados y no institucionalizados considerando la variable sociodemográfica género.

2. Describir las estrategias de afrontamiento utilizadas por los adultos mayores institucionalizados y no institucionalizados considerando la variable sociodemográfica género.

3. Describir y comparar las estrategias de afrontamiento utilizadas por los adultos mayores institucionalizados y no institucionalizados frente a la percepción de apoyo social familiar y de las amistades, considerando la variable sociodemográfica género.

Caracterización del grupo objetivo: adultos mayor de 60 años, hombres y mujeres, institucionalizados y no institucionalizados.

\section{Síntesis del marco teórico}

Es característico de esta etapa la necesidad de adaptarse a las pérdidas (de personas, físicas y de la propia muerte) (Pola, A. en Mahaluf, 1998). Frente a toda esta situación de grandes cambios, que requiere la capacidad de adaptarse a ellos, es importante la percepción que tenga el adulto mayor de este proceso y del apoyo social que posee, ya que estas variables influirán en la manera en que afronte y viva la última etapa de la vida (González, 1997).

El apoyo social se refiere a algo personal, cuyo componente más subjetivo es el apoyo social percibido. Su contrapartida socio-estructural y objetiva es la red de apoyo social, compuesta por todas las relaciones que una persona percibe como significativas. Durante la vejez coexisten tres factores con efectos acumulativos en la red social: primero se contrae, ya que el número de los vínculos se reduce, segundo, las oportunidades y la motivación para renovar la red social disminuyen; por último, los procesos para mantener la red se dificultan al disminuir la energía necesaria para mantener los vínculos activos (Sluzki, 1998).

Diversos autores plantean que el apoyo social se relaciona con la salud y calidad de vida, teniendo mayor probabilidad de enfermedad aquellas personas que les falla o carecen de apoyo social. La pérdida de éste genera una situación de estrés, potenciando la vulnerabilidad a las enfermedades, lo que moviliza las estrategias de afrontamiento (Blome, 2001). Para efectos de la presente investigación se consideraron los planteamientos de la psicología cognitiva a través de Lazarus y Folkman (1986), quienes la definen como: "Aquellos esfuerzos cognitivos y conductuales constantemente cambiantes que se desarrollan para manejar las demandas específicas externas y/o internas que son evaluadas como excedentes o desbordantes de los recursos del individuo" (Lazarus, R., Folkman, S. 1986. Pág. 164).

Un estudio realizado por Aldrich y Medkoff (1963, en Lazarus, R., Folkman, S., 1986) concluye que la forma en que el adulto mayor afronta la experiencia de pasar de una situación a otra, tiene influencia en el grado de mortalidad, mostrando que los adultos mayores que respondían en forma filosófica presentaban una tasa de mortalidad más baja que los que respondían con sentimientos de negación y depresión. Diversos estudios muestran que los adultos mayores que utilizan estrategias como la aceptación pasiva, depresión e indefensión, tienen menores expectativas de vida que los que luchan por tener el control de las circunstancias.

\section{Método:}

La presente investigación es de tipo descriptivo-comparativo, de carácter cuantitativo.

Para alcanzar estos objetivos se realizó un muestreo probabilístico estratificado, quedando la muestra compuesta por 100 adultos mayores que residen en la Región Metropolitana, los cuales se distribuyeron por género e institucionalización.

En esta investigación se utilizaron como instrumentos de recolección de datos la Escala de Apoyo Social Familiar de Procidano y Heller (1983), la Escala de Apoyo Social de las Amistades de Procidano y Heller (1983) y la Escala de Estrategias de Afrontamiento de Páez, Besabe y Valdoseda (1992). 
Para el cumplimiento de los objetivos de esta investigación se utilizaron como técnicas de análisis de los datos obtenidos: la distribución de frecuencia, la prueba " $t$ " y las técnicas no paramétricas, prueba $\mu$ de Mann-Whitney, el Coeficiente de Correlación de Spearman de rangos ordenados ( $r h o)$ y el análisis de varianza unifactorial por rangos de Kruskal-Wallis. Para los fines de esta investigación se definió como valor significativo un nivel de significacia $>$ a 0,05 .

\section{Síntesis de resultados y propuestas}

Con respecto a la percepción de apoyo social familiar, la media de la muestra es de 9.23. El $69 \%$ de los adultos mayores se encuentra sobre el promedio. En relación a la percepción de apoyo social de las amistades, la media de la muestra se ubica en 7.98 puntos. El $62 \%$ de los adultos mayores se ubica sobre la media. En relación a la percepción de ambos tipos de apoyo social, se puede apreciar que los hombres se encuentran bajo la media de la muestra y las mujeres por sobre esta. La institucionalización se constituye en una variable importante en la percepción de apoyo social tanto familiar como de las amistades, ya que los adultos mayores institucionalizados perciben menor apoyo social en comparación a los adultos mayores no institucionalizados.

En relación a las estrategias de afrontamiento se aprecian diferencias significativas entre hombres y mujeres y las estrategias tales como: reinterpretación positiva y crecimiento y en focalización y ventilación de emociones, siendo las mujeres quienes despliegan frecuentemente estas estrategias. Al comparar las estrategias de afrontamiento utilizadas por adultos mayores institucionalizados y no institucionalizados, se aprecian diferencias significativas en las estrategias: reinterpretación positiva y crecimiento, planificación, búsqueda de apoyo social por motivos instrumentales, búsqueda de apoyo social por motivos afectivos, siendo los no institucionalizados los que despliegan más estas estrategias.

Al evaluar la influencia de las estrategias de afrontamiento en relación a la percepción de apoyo social familiar en hombres, se encuentran correlaciones significativas positivas en las estrategias de afrontamiento: afrontamiento activo, planificación, búsqueda de apoyo social por motivos instrumentales y de escape y evitación.
En el caso de las mujeres se encuentra una correlación significativa positiva, en la estrategia de afrontamiento: autocontrol y contención de manera planificada. Se da también una correlación significativa negativa en las estrategias de afrontamiento: negación, de escape y evitación.

Refiriéndose a la percepción de apoyo social de amigos en hombres, se encuentran correlaciones significativas positivas, en las estrategias de afrontamiento: afrontamiento activo, búsqueda de apoyo social por motivos afectivos, focalización y ventilación de emociones. En relación a las mujeres se encuentran correlaciones significativas positivas en la estrategia de afrontamiento: búsqueda de apoyo social por motivos instrumentales. Además existe una correlación significativa negativa en la estrategia de afrontamiento: autocontrol.

$\mathrm{Al}$ establecer la influencia de las estrategias de afrontamiento respecto a la percepción de apoyo social familiar en adultos mayores institucionalizados no se aprecian correlaciones significativas. Sin embargo, con relación a la percepción de apoyo social de amigos se encuentran correlaciones significativas positivas en las estrategias de afrontamiento: autocontrol, donde existe una baja puntuación en la percepción de apoyo social de las amistades y conjuntamente se aprecia una baja utilización de la estrategia. Por lo tanto, ambas puntuaciones bajan, haciendo que la correlación se positivice.

Al determinar la influencia de las estrategias de afrontamiento con relación a la percepción de apoyo social familiar en adultos mayores no institucionalizados, se encuentran correlaciones significativas positivas en las estrategias de afrontamiento: autocontrol y contención de manera planificada, reinterpretación positiva y crecimiento. Además se encuentra una correlación significativa negativa con las estrategias de afrontamiento: negación y descompromiso mental. Se pueden establecer respecto a la percepción de apoyo social de amigos, correlaciones significativas positivas en las estrategias de afrontamiento: búsqueda de apoyo social por motivos instrumentales, búsqueda de apoyo social por motivos afectivos y la focalización y ventilación de emociones. Además se encuentra una correlación negativa significativa con la estrategia de afrontamiento: autocontrol.

Se podría hipotetizar que en la adultez mayor el género no es una variable determinante 
en los procesos vividos como en las anteriores etapas del ciclo vital, debido a que los elementos negativos relacionados con la vejez en las representaciones sociales compartidas no discriminan entre los hombres y mujeres adultos mayores (llamada Viejismo).

La variable institucionalización marca una significativa diferencia, lo que podría relacionarse con las consecuencias de este proceso, ya que los adultos mayores dejan de tener una vida independiente, en la cual se limita el contacto con el mundo exterior y se pierde la privacidad (Sánchez, E., 1990). Del mismo modo se pierde el contacto con las redes de apoyo primarias, mostrando los adultos mayores una escasa motivación para renovarlo (Sluzki, 1998).

Según los resultados, se aprecia que los adultos mayores utilizan frecuentemente los métodos de afrontamiento activo para enfrentar el estrés, atendiendo a la situación estresante y empleando recursos cognitivos-conductuales para lograr un cambio frente al problema que puedan estar atravesando. Tal como lo ha planteado Reeve (1994) y como lo ha señalado Barros (1999), buscan una solución frente a lo que acontece, solicitan ayuda, apoyo, es decir, utilizan estrategias dirigidas al problema, intentando manejar lo que genera el estrés, definiendo la situación, aplicando posibles soluciones. Es importante señalar que estos hallazgos se contraponen a lo planteado por Zapata (2001) y Gutmann (1979), quienes caracterizan la participación de los adultos mayores como una recepción de las acciones más que la generación de tareas propias, relacionado con una representación social de connotación negativa, que muchas veces lleva a discriminarlos, limitando sus oportunidades en esta sociedad. Lo anterior nos obliga a revisar la concepción de la adultez mayor, centrándose no sólo en las pérdidas sino también considerando sus propios recursos.

Se puede apreciar que cuando los adultos mayores perciben mayor apoyo social familiar despliegan estrategias más activas, considerándolo como un recurso positivo que les permite afrontar los retos del ambiente de mejor mane$\mathrm{ra}$, siendo el apoyo un moderador frente a los acontecimientos estresantes (Martínez, M. et al., 2001). Además el contar con amigos, le permite al adulto mayor satisfacer necesidades fuera del núcleo familiar, sin que se replieguen en este núcleo sobrecargándolo (Sluzki, 1998).
A partir de los resultados obtenidos se podría hipotetizar que al percibir las mujeres adultas mayores mayor apoyo social se benefician de las características de éste y protectoras en la salud, que les permite atenuar o amortiguar los efectos del estrés, los cuales debilitan la salud del organismo. A su vez, utilizan estrategias de afrontamiento más activas, realizando acciones que apuntan a enfrentar la situación y eliminar al estresor. Los aspectos antes mencionados contribuirían a que las mujeres tengan mayores expectativas de vida, éstas se mantendrían más saludables pues resignificarían de manera más positiva y activa las situaciones de estrés, con lo que lograrían tener una mejor salud y vivir más años que los hombres.

En la presente investigación se concluyó que no es la edad la variable determinante de la utilización de las estrategias de afrontamiento, sino la evaluación secundaria que realiza el sujeto, donde la percepción de apoyo social cumple un rol fundamental como recurso. Esto queda de manifiesto en el hecho de que los adultos mayores institucionalizados perciben menor apoyo social; tienen un espectro de utilización de estrategias más reducidas, siendo estas principalmente evitativas o pasivas y finalmente no se encuentran correlaciones significativas al considerar las variables percepción de apoyo social (familiar y de las amistades) y las estrategias de afrontamiento. Por lo tanto, al realizar la evaluación secundaria de la situación estresante, no consideran dentro de sus recursos para la solución del evento estresante a la familia ni a las amistades, pudiendo ser este el motivo de que no existan correlaciones entre estas dos variables.

Si bien el tema del adulto mayor es un área que en el último tiempo ha tenido gran auge, no existen investigaciones acerca de las estrategias de afrontamiento y la percepción de apoyo social. El conocerla permitirá trabajar potenciando las estrategias de afrontamiento adaptativas para que, dentro de esta vivencia que significa la vejez, se puedan distinguir recursos. Todo lo anterior con la finalidad de mejorar la calidad de vida del adulto mayor en Chile.

\section{Bibliografia}

ABARCA P., HUMBERTO. (2000). Discontinuidades en el modelo hegemónico de masculinidad. Red de Masculinidad. Facultad Latinoamericana de Ciencias Sociales (FLACSO). Chile. 
ARANGO, L.; LEÓN, M.; VIVEROS, M. (1995). Género e Identidad. Ensayos sobre lo femenino y lo masculino. Tercer Mundo editores. Ediciones Uniandes. Bogotá.

ARBINAGA I., FÉLIX. (2002). La mujer institucionalizada $v / s$ no institucionalizada: aproximaciones al estado de ánimo, la ansiedad ante la muerte y su satisfacción ante la vida. Cuaderno de Medicina Psicosomática y Psiquiatria de Enlace (C.MED.PSICOSOM). N ${ }^{\circ} 64$.

ARON, A.; NITSCHE, R.; ROSENBLUTH, A. (1995). Redes sociales de adolescentes: un estudio descriptivo comparativo. Revista PSYKHE. Volumen 4. $\mathrm{N}^{\circ} 1$. Pontificia Universidad Católica de Chile. Chile.

BARRÓN L., ANA; SÁNCHEZ M., ESTEBAN. (2001). Estructura social, apoyo social y salud mental. Universidad Complutense de Madrid. Psicothema. CODEN PSOTEG 2001. Volumen 13. $N^{\circ} 1$. España.

BARROS, CARMEN. (1996). Trabajando hacia un envejecer pleno. (Taller de entrenamiento en resolución de problemas y aceptación de pérdidas). Cuadernos del Instituto de Sociología. Pontificia Universidad Católica de Chile. Chile.

BARROS, CARMEN. (1999). Estudios sociales $N^{\circ} 100$. Trimestre 2. Corporación de Promoción Universitaria (CPU). Chile.

BERGER, C.; MILICIA, N.; ALCALAY, L.; TORRETTI, A. (2002). Ser padres de adolescentes hoy: un programa audiovisual para reflexionar en torno a la socialización de género en el contexto familiar. Revista PSYKHE. Volumen 11. $\mathrm{N}^{\circ} 1$. Pontificia Universidad Católica de Chile. Chile.

BIRGIN, HAYDEÉ; PAUTÁIS, LAURA. (2001). Género en la reforma o reforma sin género? Desprotección social en las leyes provisionales de América Latina. Serie Mujer y Desarrollo $N^{\circ} 36$. Comisión Económica para América Latina y el Caribe (CEPAL). Chile.

BLOME B., CRISTIAN. (2001). Estrategias de afrontamiento en guardias de seguridad con alto y bajo rendimiento en instalaciones de riesgo. Tesis para optar al titulo de Psicólogo. Universidad Santo Tomás. Chile.

BRAVO, CAROL; CARO, MARCELA. (2002). Efectos psicosociales de la jubilación por vejez en las variables de autoestima y apoyo social en el adulto mayor afiliado al Instituto de Normalización Provisional (INP) de la Región Metropolitana. Revista PSYKHE. Volumen 11. $\mathrm{N}^{\circ}$ 2. Pontificia Universidad Católica de Chile. Chile.

CARRETERO, M.; PALACIOS, J.; MARCHESI, A. (1997). Psicología Evolutiva: adolescencia, madurez y senectud. Editorial Alianza Psicología. España.

CARRIÓN, A.; CÓRDOVA, A. (2003). Diferencias en la percepción de influencia de los acontecimientos vitales en hombres y mujeres. Revista Latinoamericana de Psicología. Volumen $35 . \mathrm{N}^{\circ} 1$. Colombia.

CONDE SALAS, L (2001). Vivir la vejez positivamente. Primer curso virtual de educación para el envejecimiento. http://www.psiconet.com/tiempo/educacion http:// www.psiconet.com/tiempoleducacion.

DONOSO, I.; HERRERA, C.; SALINAS, C. (1999). Intervención social gerontológico. Una experiencia descriptiva del fenómeno del adulto mayor institucionalizado en el marco de la primera experiencia local de trabajo en redes. Tesis para optar al Grado de Licenciado en Trabajo Social y al Título profesional de Asistente Social. Universidad Santo Tomás. Chile.
DULCEY-RUIZ, E.; URIBE, C. (2002). Psicología del ciclo vital: hacia una visión comprehensiva de la vida humana. Revista Latinoamericana de Psicología. Volumen 34. $N^{\circ} 1-2$. Colombia.

DUQUE, Ma DEL PILAR (2002). Representaciones sociales de roles de género en la yejez: una comparación transcultural. Revista Latinoamericana de Psicologia. Volumen 34. Colombia.

ERIKSON, E. (1985). El ciclo vital completado. Editorial Paidos. México.

ESCOBAR, D.; FLORES, R.; VENEROS, D. (2001). Investigaciones Red Nacional Universitaria Interdisciplinaria de estudios de género. Servicio Nacional de la Mujer (SERNAM). Chile.

FORTTES, A. (1995). Los nuevos desafios del retiro laboral. Revista de Trabajo Social. $N^{\circ} 65$. Pontificia Universidad Católica de Chile. Chile.

FOSIS. (1996). Programa del adulto mayor: Más vida para tus años. Gobierno de Chile. Chile.

GIDDENS, ANTHONY. (1995). Sociologia. Editorial Alianza. España.

GOMÁRIZ MORAGA, ENRIQUE. (1992). Los estudios de género y sus fuentes espistemológicas: periodización y perspectivas. Serie de estudios sociales $N^{0} 38$. Facultad Latinoamericana de Ciencias Sociales (FLACSO). Chile.

GONZÁLEZ B., CAROLINA; LAVÍN F., CAROLINA; MARTINEZ D., LUIS. (1997). Resiliencia y Adultez Mayor: construyendo en adversidad. Tesis para optar al Grado de Licenciado en Servicio Social y al Título de Asistente Social. Universidad Santo Tomás. Chile.

GONZALEZ GADYS. (2003). Perfil del adulto mayor y su comunidad. Servicio Nacional del Adulto Mayor. Chile.

HERNÁNDEZ S., ROBERTO; FERNÁNDEZ C., CARLOS; BAPTISTA L., PILAR. (1998). Metodología de la investigación. Editorial Mc Graw Hill. México.

INSTITUTO NACIONAL DE ESTADÍSTICAS (INE). (2003). Resultados del Censo 2002. Gobierno de Chile. Chile.

INSTITUTO DE NUTRICIÓN Y TECNOLOGÍA DE LOS ALIMENTOS (INTA). (1996). Clasificación funcional de adultos mayores a nivel de comunas prioritarias Chile 1995. Chile.

IZAL, MARÍA; MONTORIO, IGNACIO. (1999). Gerontología conductual: bases para la intervención y ámbitos de aplicación. Editorial Síntesis. España.

KORNFELD, R.; MARÍN, P.; ORELLANA, V. (2000). Mitos y modelos de envejecimiento activo. Colección Adulto Mayor. Pontificia Universidad Católica de Chile. Chile.

LAFOREST, JACQUES. (1991). Introducción a la gerontología. El arte de envejecer. Editorial Herder. España.

LAGARDE, MARCELA. (1992). Identidad de Género. Cuaderno de trabajo. Centro para la participación democrática y el desarrollo. Cenzotie. Nicaragua.

LAZARUS S, RICHARD; FOLKMAN, SUSAN. (1986). Estrés y procesos cognitivos. Ediciones Martínez Roca S.A.. España.

LEHR, URSULA. (1995). Psicologia de la senectud. Editorial Herder. Barcelona.

MAHALUF, J.; NADER, A.; KORNFELD, R. (1998). Reflexiones psicológicas para el adulto mayor: Colección Adulto Mayor. Pontificia Universidad Católica de Chile. Chile. 
MARÍN, PEDRO. (1993). Tiempo nuevo para el adulto mayor: Enfoque interdisciplinario. Colección Adulto Mayor. Programa para el Adulto Mayor. Pontificia Universidad Católica de Chile. Chile

MARTÍN G., ANTONIO; CHACÓN F., FERNANDO; MARTÍNEZ G., MANUEL. (1988). Psicología Comunitaria. Textos Visor. España.

MARTÍNEZ, M.; GARCÍA, M.; MAYA, I. (2001). El efecto amortiguador del apoyo social sobre la depresión en un colectivo de inmigrantes. Universidad de Sevilla. Psicothema. CODEN PSOTEG 2001. Volumen $13 \mathrm{~N}^{\circ} 4$. España.

MILAGROS, MARTÍN. (2001). Envejecimiento y cambios psicológicos. Trabajo monográfico para el curso virtual Educación para el envejecimiento. http://psiconet.com/ tiempo http://psiconet.com/tiempo.

MINISTERIO DE PLANIFICACIÓN Y COOPERACIÓN (MIDEPLAN). (1999). Resultados Encuesta CASEN 1998, Documento $N^{\circ}$ 10: Situación de los adultos mayores en Chile. Chile.

MINISTERIO DE PLANIFICACIÓN Y COOPERACIÓN (MIDEPLAN). (2000). Resultados de la VIII Encuesta CASEN 2000, Documento No 12: Situación de los adultos mayores en Chile. Chile.

MONCHIETTI, A.; KRZEMIEN, D. (2000). Participación social y estilo de vida. Su relación con la calidad de vida en la vejez. Revista Tiempo de Psicogerontología $\mathrm{N}^{\circ} 6$. http://psiconet.com/tiempo http://psiconet.com/tiempo.

MYERS, DAVID. (2000). Psicología Social. Editorial Mc Graw Hill. Bogotá

PAPALIA, D., WENDKOS, S. (2001). Desarrollo Humano. Editorial Mc Graw Hill. México.

PONCE A., CLAUDIA. (2001). Burnout y estrategias de afrontamiento en profesores del segundo ciclo de enseñanza básica de los establecimientos municipales de la comuna de San Joaquín. Tesis para optar al Grado Académico de Magister en Psicología mención Educacional. Pontificia Universidad Católica de Chile. Chile.

PONCE A., CLAUDIA. (2002). Burnout y estrategias de afrontamiento en profesores educación básica. Revista PSYKHE . Volumen 11. $\mathrm{N}^{\circ}$ 2. Pontificia Universidad Católica de Chile. Chile.

REEVE, JOHN. (1994). Motivación y emoción. Editorial McGraw - Hill. España.

RODRÍGUEZ MARÍN, JESÚS (1995). Psicología Social de la salud. Editorial Síntesis S.A. España.

SÁNCHEZ SALGADO, CARMEN. (1990). Trabajo social $y$ vejez. Editorial Humanitas. Argentina.
SÁNCHEZ VIDAL, ALIPIO. Psicología Comunitaria. Bases conceptuales y métodos de intervención. $1^{\circ}$ Edición. Editorial EUB, S.L. España.

SIEGEL, S.; CASTELLAN, N. (1998). Estadistica No Paramétrica aplicada a las ciencias de la conducta. Editorial Trillas. México.

SILVA M., MARCELO. (2000). Estudio comparativo de las variables Psicosociales: estrés percibido, estrategias de afrontamiento y apoyo social entre ejecutivos y administrativos de Bancos Comerciales de la Región Metropolitana. Tesis para optar al título profesional de Psicólogo. Universidad Santo Tomás. Chile.

SILVESTRE, N.; SOLÉ, M.; PÉREZ, M.; JODAR, M. (1995). Psicología Evolutiva : adolescencia, edad adulta y vejez. Colección educación y enseñanza. Serie Universitaria. Ediciones CEAC. España.

SLUZKI, CARLOS. (1998). La red social: frontera de la práctica sistémica. Editorial Gedisa S.A. España.

SOSA, ZULMA; HUENCHUAN, SANDRA. (2002). Redes de apoyo social de personas mayores en Chile. Consultoras CELADE - División de población de la CEPAL. Chile.

SPECK, ROSS; ATTNEAVE, CAROLYN. (2000). Redes familiares. Editorial Amorrortu. Argentina.

STEINBERG, LEA. (2002). Acerca de los adultos mayores. Actualidad Psicológica. Periódico mensual. Año XXVII. N ${ }^{\circ}$ 301. Buenos Aires. Argentina

SUBIRATS, JOAN. (1992). La vejez como oportunidad para crecer: Instituto Nacional de Servicio Sociales. Ministerio de Asuntos Sociales. España.

TAMER, NORMA. (1995). El envejecimiento humano. Sus derivaciones pedagógicas. Colección Interamer 51 . Serie educativa. Estados Unidos.

THOMAE, HANS. (2002). Haciéndole frente al estrés en la vejez. Revista Latinoamericana de Psicologia. Volumen 34. Colombia.

WOOD, PHILIP Y VERA, ALDO. (1994). Un modelo explicativo de salud mental basado en categorias psicosociales: Descripción teórica de sus componentes, construcción de una batería de instrumentos para la evaluación de factores psicosociales y prueba empirica del modelo. Tesis para optar al título de Psicólogo. Universidad Diego Portales. Chile.

ZAPATA F., HERNÁN. (2001). Adulto mayor: participación e identidad. Revista de Psicología. Departamento de Psicologia Facultad de Ciencias Sociales Universidad de Chile. Vol. X N ${ }^{\circ} 1$. Chile. 